In the case of informed consent it is not your

responsibility to listen and understand, it is my

responsibility to ensure you have heard and

understood.

\title{
Importance of consent
}

The research paper by Tahir, Mason and Hind on Informed consent: optimism versus reality (page 221) in this issue raises some interesting questions about how successfully dentists communicate with patients when it comes to informed consent. In fact, one might be forgiven for thinking that informed consent has been 'done to death' in the dental literature. Surely people understand it by now, but this paper suggests otherwise.

The details of the paper are that the parents of children requiring extractions at The Eastman Dental Institute in London were interviewed and asked to complete a structured questionnaire just after they had signed a consent form to the treatment and a second time just before the treatment was carried out. The explanation of the treatment required was provided in a consistent manner and observed by one of the researchers to ensure all the information was provided. Despite this the research showed that 40\% of the written consent obtained was not valid, and while the parents' understanding appeared to improve on the actual day of treatment 19\% still did not know exactly what was going to happen to their children just before the treatment was carried out.

At first sight the figure of 40\% seems worryingly high. Over one third of parents in the study were apparently signing consent forms without truly appreciating what they were signing or what was going to happen to their children. But further reflection begins to question whether this finding is so surprising after all. We need to remember that the situation is fairly stressful for parents and most of us have been in situations far less stressful where we have found it immensely difficult to understand exactly what is going to happen.

For example, when I take my car for servicing the explanation for what has been done is incomprehensible (to me) despite a careful and detailed description and invoice. I found that after a recent visit to my solicitor to update my will, on the way home I was still not sure exactly what had been discussed, despite a very good explanation at the time. I thought I knew what was happening, but I doubt very much I could have explained it to someone else afterwards, or confidently completed a questionnaire for a research study. These examples simply reveal how difficult it is for us to understand simple matters that are totally outside our normal experience, even when the explanation is done very well.

The other matter I reflected on after reading the paper was whether the parents would have been better or worse at understanding the explanation in a general practice environment. One might have assumed not, given that a hospital environment does not suffer from the commercial pressures of practice, and thus would presumably have more time for explanation. But that way of thinking is both simplistic and naïve. Casualty departments have different time pressures (often far worse than NHS general practice), the house officer is a stranger to the parents, the whole hospital environment can be very stressful for patients and so on. It would be interesting to follow this research with a similar study in a general practice to see the results before we jump to any conclusions.

So what can we infer from this study? Despite my reflections above the study is relevant to us all, because it highlights yet again how important it is to ensure that people are absorbing and understanding treatment recommendations and advice. It is no good assuming because I am telling you something that you are hearing it. And in the case of informed consent it is not your responsibility to listen and understand, it is my responsibility to ensure you have heard and understood. Thus skill in effective communication is vital, and despite the growing emphasis in dental education on this skill perhaps we need even more. We definitely need more help post qualification, and help from experts trained in providing skills training rather than enthusiastic and interested dentists. In essence, we need to be taking communication skills training really seriously, perhaps as seriously as we take clinical training.

Yet there is one ray of real sunshine in this paper which I found both reassuring and uplifting. Despite the fact that $40 \%$ of people did not understand what was going to happen to their children the majority continued with the treatment, putting their trust in the hospital and the clinicians to make their children well again. That really is a welcome note to end on.

Mike Grace m.grace@bda-dentistry.org.uk 